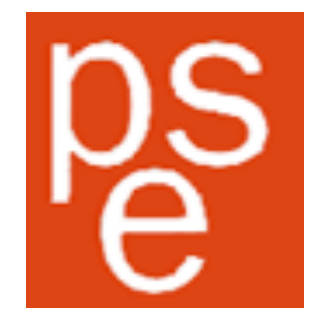

\title{
La crise a-t-elle rendu l'épargnant plus prudent ?
}

\author{
Luc Arrondel \\ CNRS-PSE et Banque de France
}

André Masson

CNRS-PSE et EHESS

Septembre 2010

\footnotetext{
L'enquête utilisée dans cette étude a bénéficié du soutien financier du Cepremap, de la Chaire "Les particuliers face au risque" (Fondation du Risque-Groupama), de l'Institut CDC pour la Recherche et de l'Institut Europlace de Finance. Les auteurs remercient également les participants au séminaire scientifique de la Cdc Retraite à Bordeaux (mars 2010) pour leurs remarques.
} 
"The subprime crisis along with its associated financial and economic problems is due, in good measure, to some failures of democracy-financial democracy, that is. Many working-class people and first-time home buyers who took out high loan-tovalue mortgages with adjustable rates did not have ready access to information about what they were doing - the kind of information easily available to wealthier people - and so made serious mistakes."

Robert Shiller, October 2008

\section{I - INTRODUCTION}

La crise économique et financière actuelle initiée en 2007 a connu différentes étapes marquées par plusieurs temps forts : crise dite des «subprimes » durant l'été 2007 après le dégonflement de la bulle immobilière américaine des années 2000, effondrement des marchés boursiers internationaux à l'automne 2008 faisant suite aux faillites de plusieurs établissements financiers américains, creusement des déficits publics dans de nombreux pays en 2009, les finances publiques ayant été fortement sollicitées par les gouvernements pour atténuer les effets de la récession.

En France, la crise financière et immobilière a vu la bourse chuter de plus de $40 \%$ et le prix des logements d'environ 7\%. Selon les données de la comptabilité Nationale (Insee), elle aurait provoqué une baisse de la richesse brute des ménages français entre 2007 et 2008 de $3 \%$ au niveau macroéconomique ; la diminution correspondante serait de 3,7\% pour la richesse nette et de 5,6\% seulement pour le patrimoine financier, en raison notamment de la faible diffusion des actifs boursiers dans la population (seulement $15 \%$ d'actionnaires directs). Le patrimoine moyen, lui, aurait enregistré une baisse de 4,2 \%, correspondant à un montant de près de 16400 euros par ménage, dont plus de 9200 euros pour les seules pertes financières.

Pour mémoire, notons que ces pertes ont été très inférieures à celle observées ailleurs, notamment aux États-Unis et au Royaume-Uni. Ainsi aux États-Unis, selon les chiffres de la Federal Reserve, la baisse de la richesse brute des ménages américains en 2008 se situerait globalement aux environs de $17 \%$ (20\% en richesse nette et $18 \%$ en richesse financière), la fortune moyenne ayant diminué de près de $18 \%$, soit une diminution du patrimoine de chaque américain de plus de 100000 dollars. Au Royaume-Uni, selon les données de l'Office for National Statistics, les ménages Britanniques auraient perdu près de $10 \%$ de leur richesse brute (12\% de richesse nette et $10 \%$ de leur richesse financière), le patrimoine moyen ayant perdu $10,5 \%$ de sa valeur ${ }^{1}$. En 2009, toutefois, la situation patrimoniale des ménages s'est améliorée à des degrés divers dans ces trois pays : $+0,8 \%$ de richesse brute en

\footnotetext{
${ }^{1}$ Les ménages allemands ont cependant été moins touchés, ne perdant en moyenne que 3100 euros.
} 
France $(+8 \%$ pour les actifs financiers), $+3,5 \%$ aux États-Unis (respectivement $+6,8 \%$ ) et $+8 \%$ au Royaume-Uni (respectivement $+12,8 \%$ ).

Comment l'épargnant a-t-il réagi à cette crise, face aux aléas boursiers, au durcissement des conditions de crédit et aux anticipations de baisse des prix des logements, aux perspectives économiques alarmistes relatives au pouvoir d'achat et au chômage ? Le taux d'épargne des fourmis françaises est resté à des niveaux élevés (autour de $15 \%$ qui est son niveau depuis une quinzaine d'années et de $5 \%$ pour le financier) et a même augmenté en 2009 pour atteindre $16,3 \%$ du revenu disponible brut des ménages $(6,8 \%$ pour l'épargne financière). Parallèlement, la croissance de la consommation qui oscillait autour de $4 \%$ depuis 2008 a fortement décru en 2008 où elle plafonnait à $0,1 \%$. Entre 2007 et 2009, le taux d'épargne a de fait augmenté presque partout en Europe, surtout dans les pays les plus touchés (Oecd, 2010) : il est passé de 2,2 \% à $7 \%$ au Royaume-Uni, de 10,6\% à 18,8 \% en Espagne et s'est maintenu en Allemagne et en Italie ; ce taux est passé de 1,7\% à 4,3\% du revenu disponible des ménages américains. Comment l'épargnant a-t-il «assimilé » ces évolutions ?

Selon l'Ocde (Oecd, 2010), tous les indicateurs liés à la confiance des consommateurs européens et américains montrent une chute générale «d'optimisme »après l'été 2007 (crise des subprime) jusqu'à la fin 2008, la légère amélioration de l'année 2009 étant sans doute en train de s'essouffler : autrement dit, l'augmentation de l'épargne proviendrait d'un désir de précaution accru et/ou d'effets de richesse (subjective) négatifs. Les effets de richesse auraient été beaucoup plus importants aux États-Unis et au Royaume-Uni où les ménages pouvaient plus facilement gager leurs emprunts sur leurs avoirs et où les retraites sont principalement financées par l'épargne privée: selon une étude de l'Insee (Lapègue et Mauroux, 2010), l'impact de la dévalorisation des avoirs patrimoniaux aurait coûté 1,7 point de croissance du PIB aux USA, 0,9 au Royaume-Uni alors que dans la zone euro, l'impact n'aurait été que de 0,4 point (et 0,2 seulement en France et en Allemagne).

En France, plus particulièrement, la plupart des indicateurs macroéconomiques, mais également les enquêtes d'opinions, montreraient que les comportements des ménages sont devenus plus prudents et plus prévoyants, la tendance étant de vouloir s'éloigner des investissements boursiers pour privilégier les placements plus sûrs mais aussi de plus long terme (assurance vie et épargne logement). Encore en 2009, une hausse de la consommation $(0,8 \%)$ inférieure à celle des revenus (4\%) attesterait l'impact des effets de richesse. Mais ces derniers ne sont sans doute pas homogènes dans la population, en particulier en fonction du niveau de fortune. Ainsi, Le Figaro du 10 juin 2009 titrait "En temps de crise, les riches s'amusent encore plus" montrant qu'en matière d'activités de loisirs de luxe (restaurants, voyages, concerts et spectacles), les réservations avaient augmenté de $40 \%$ au début de 2009 juste après la chute des bourses mondiales! En particulier, le nombre de voyages a été 
multiplié par trois au cours des six premiers mois de 2009, même si "... les séjours d'une semaine ont laissé place à de longs week-ends de quatre jours, avec un budget moyen de 2450 euros au lieu de 4050 euros au second trimestre $2008 " .$.

Les donnés précédentes, macro-économiques, qualitatives ou subjectives ne permettent pas cependant de rendre compte de cette hétérogénéité des comportements et des réactions face à la crise. Surtout, question clef pour le micro-économiste, elles ne permettent pas d'en déterminer l'origine : ces changements de comportements, pour autant qu'ils puissent être plus précisément cernés, sont-ils dus seulement à une réaction face au contexte économique de crise ou les préférences individuelles en matière de risque et vis-à-vis de l'avenir se sontelles mêmes modifiées, les ménages étant devenus d'une part, plus averses au risque ou plus prudents et d'autre part plus prévoyants ou plus soucieux de leur futur? Autrement dit, les préférences de l'épargnant sont-elles soumises aux aléas de la conjoncture financière?

Le choc exogène de la crise financière, sorte "d'expérience naturelle », permet ainsi de tester une des pierres angulaires de la théorie économique, à savoir la stabilité temporelle des préférences. L'intérêt de notre étude dépasse néanmoins le cadre académique puisque les conclusions que l'on peut en tirer concernent également le débat politique actuel et les projets de réforme concernant la retraite, la santé, ou la dépendance : la volonté de certains visant à responsabiliser davantage les ménages pour suppléer le désengagement éventuel de l'État a mis sous les projecteurs les comportements de l'épargnant en général et sa rationalité en particulier.

Nous disposons d'une source statistique unique pour répondre à ces différentes questions (section II). Cette dernière est constituée notamment par les enquêtes Tns-Sofres effectuées sous notre initiative notamment en mai 2007 (avant la crise) et juin 2009 auprès de 3800 ménages français représentatifs, avec une importante dimension de panel : plus de 2200 ménages de l'enquête 2007 ont été réinterrogés en 2009. Ces données individuelles, particulièrement riches, révèlent effectivement, chez une majorité de ménages, une moindre volonté de prendre des risques en matière financière depuis la crise, ainsi qu'une plus grande prévoyance (section III).

La question centrale de cet article consiste à expliquer ce changement de comportement : la crise a-t-elle changé l'épargnant, ou seulement l'environnement économique global et sa situation personnelle, telle qu'il la perçoit (baisse anticipée des ressources ou des rendements d'actifs, exposition au risque plus élevée) ? Pour répondre, on doit disposer de mesures directes des préférences individuelles face au risque et au temps: les enquêtes nous permettent de comparer les mesures conventionnelles, disponibles dans la littérature (basées sur des choix hypothétiques de loterie ou échelles subjectives, par exemple), à nos propres mesures, obtenues par une méthode extensive et originale de scoring : le score de risque est 
ainsi établi à partir des réponses fournies à une soixantaine de questions très diverses. L'introduction de scores est justifiée par des performances bien supérieures aux autres mesures, tant en matière de robustesse (déterminants individuels des préférences, effets de ces dernières sur le patrimoine, etc.), d'exogénéité (par rapport aux comportements patrimoniaux à expliquer) ou de corrélation au cours du temps obtenue sur données de panel (section IV). Comment ces indicateurs de préférence ont-ils évolués entre mai 2007 et juin 2009 pour les mêmes individus (« panélisés ») ?

Les mesures conventionnelles concluent toutes à une moindre tolérance au risque et à une plus grande prévoyance, alors que les distributions de nos scores d'attitude à l'égard du risque et de préférence temporelle sont quasiment identiques. Au vu de la supériorité de notre méthode, nous concluons que les préférences des épargnants français à l'égard du risque et du temps sont restées jusqu'ici stables en moyenne pendant la crise, ce qui est une bonne nouvelle tant pour la théorie micro-économique que pour la pratique standard qui ignore les évolutions d'éléments inobservables comme c'est le cas, en général, des préférences individuelles (section $\mathrm{V}$ ). Ce résultat ne préjuge pas, cependant, des évolutions ultérieures possibles en France $^{2}$, ni de l'existence d'un scénario différent dans d'autres pays où notre méthode de scoring pourrait être appliquée.

\section{II - COMMENT SAVOIR SI L'EPARgNANT EST DEVENU PLUS PRUDENT OU PLUS PREVOYANT ?}

Fruit d'une expérience de plus dix années, notre corpus de données est constitué de quatre enquêtes successives ayant pour objectif principal d'étudier parallèlement les patrimoines des ménages et leurs préférences en matière d'épargne (tolérance au risque, préférence pour le présent, altruisme...).

Des données d'enquête uniques : Tns-Sofres mai 2007-juin 2009

La première enquête a été réalisée par l'Insee en 1998 (Arrondel et al., 2004). et constituait un module complémentaire à l'enquête "Patrimoine 1998", sous la forme d'une seconde interview menée auprès de 1135 ménages. Les trois autres ont été construites à notre initiative par Tns-Sofres, sous la forme d'un questionnaire postal : en 2002 sur un échantillon de 2460 ménages âgés de 35 à 55 ans ${ }^{3}$; en 2007 sur 3826 ménages et en 2009 sur 3783 ménages représentatifs de la population française.

\footnotetext{
${ }^{2}$ En effet, si un tel choc conjoncturel était amené à durer, les préférences pourraient être affectées. C'est ce qui différencie les effets de génération de simples effets de moment : un effet de génération est un effet de moment qui prend de l'âge...

${ }^{3}$ L'objectif principal de cette enquête étant aussi d'interroger, à partir du même questionnaire, deux générations co-existantes, l'échantillon principal ne retenait que des individus âgés de 35-55 ans, générations les plus à même d'avoir un enfant indépendant ou un parent vivant. Nous avons pu ainsi étudier la transmission intergénérationnelle des préférences (Arrondel et Masson, 2007).
} 
Les deux dernières enquêtes comportent les éléments pertinents pour permettre une analyse fine des réactions des épargnants français à la crise financière. Celle de mai 2007 fournit en effet un état des lieux juste avant la crise ; en outre, une part important de l'échantillon d'alors, soit 2234 ménages, a été réinterrogée en juin 2009. Cette dimension de Panel s'étend même à certains ménages enquêtés en 2002, soit juste après «l'éclatement de la bulle internet ». Par ailleurs, l'enquête de 2009 comprend tout un module sur les réactions des ménages à la crise.

L'information recueillie dans les différentes enquêtes concerne déjà la description sociodémographique du ménage, l'évaluation, la composition et le mode de gestion de son patrimoine, le recensement de ses revenus, l'existence de transferts intergénérationnels reçus ou versés, mais aussi des données plus subjectives concernant les anticipations de croissance et de risque (à 5 ans par exemple) sur le revenu personnel et les prix d'actifs.

\section{Des informations originales sur les préférences individuelles}

Les quatre enquêtes comprennent un grand nombre de questions plus qualitatives visant à mesurer les préférences individuelles à l'égard du risque et du temps à partir de choix de loteries, mais aussi en fonction des attitudes, des opinions, des comportements dans différents domaines de la vie (santé, professionnel, loisirs, famille, consommation, retraite...).

L'enquête «patrimoine » 1998 de l'Insee et les enquêtes Tns-Sofres de 2002 et 2007 nous ont permis de mettre au point, à partir de toutes ces questions, une méthodologie pour mesurer les préférences des épargnants vis-à-vis du risque et du temps (Arrondel et Masson, 2008). Cette méthode éprouvée sur les différentes données propose des mesures ordinales synthétiques, sous forme de scores, des attitudes vis-à-vis du risque des individus, de leur préférence pour le présent, et de leur altruisme familial. La multiplication des données nous a permis de vérifier que ces indicateurs sont particulièrement robustes (par rapport au type de questions retenues, aux caractéristiques et aux facteurs explicatifs de ses scores, de leurs corrélations et de leurs effets sur le patrimoine, etc.). Les scores possèdent par ailleurs des propriétés en général plus satisfaisantes que les mesures de préférences conventionnelles obtenues par exemple à partir de choix hypothétiques de loteries ou d'échelles auto-déclarées (Arrondel et Masson, 2008).

La nouvelle enquête de 2009 permet d'aller encore plus loin dans la compréhension des comportements patrimoniaux en mettant notamment en parallèle l'évolution des préférences au cours du temps en situation de crise et celle des patrimoines détenus. En particulier, pour les ménages ayant déjà répondu en 2007, les données vont nous permettre de savoir si les changements de comportement sont dus à des modifications des préférences des agents ou 
bien s'il s'agit plus simplement d'une adaptation au nouvel environnement économique et financier (baisse des ressources, exposition accrue aux risques et révisions des anticipations).

\section{III - LES REACTIONS DES EPARGNANTS FRANÇAIS A LA CRISE}

Nous analysons ici les réactions des épargnants Français face à la crise financière. Les informations, à un niveau individuel, sont issues des enquêtes Tns-Sofres réalisée en 2007 et 2009 sur des échantillons de 3800 ménages représentatifs de la population Française,

\section{Les ménages prennent moins de risque dans leurs choix financiers}

La distribution de la population en fonction du niveau de richesse financière montre peu de changement avant et après la crise financière de 2008 : pour les ménages ayant répondu aux deux enquêtes, seuls les plus riches (plus de 150000 euros) ont vu leur nombre augmenter. Parmi les investisseurs en bourse les mouvements ont été plus marqués : on dénombre plus de petits détenteurs (moins de 15000 euros) mais aussi plus de gros détenteurs (plus de 150000 euros), signe d'un accroissement des inégalités parmi les actionnaires sur la période.

Ces évolutions sont confirmées par les sentiments des ménages eux-mêmes quant à leurs avoirs patrimoniaux : un tiers estime que son patrimoine financier a diminué pendant la crise mais $6 \%$ d'entre eux considèrent qu'il a augmenté malgré la conjoncture, le reste jugeant qu'il n'a pas été affecté. Le verdict est encore plus net chez les actionnaires : les trois-quarts déclarent que leur portefeuille a baissé et un sur dix n'a vu aucune évolution, mais ils sont néanmoins 4,5\% à avoir vu leurs titres prendre de la valeur. Le constat sur l'estimation de la résidence principale est plus partagé : ils sont aussi nombreux à avoir vu leur maison prendre de la valeur qu'en perdre (40\%), les autres (20\%) ne voyant aucun changement.

L'enquête Tns-Sofres 2009 fournissait aussi certaines informations subjectives sur le vécu de la crise. A la question «Aujourd'hui, diriez-vous que la crise vous concerne personnellement », la moitié des ménages répondent qu'ils se sentent beaucoup ou assez concerné, surtout des ménages âgés de 40 à 60 ans, n'ayant pas de hauts diplômes et disposant de faibles ressources (en patrimoine et en revenu). L'autre moitié qui surfe sur la crise concerne plutôt les jeunes (moins de 30 ans) et les vieux retraités (plus de 70 ans), fortement diplômés (troisième cycle ou Grandes écoles), ayant de fortes ressources patrimoniales (au moins 30000 euros de richesse financière) et salariales (supérieures à 2700 euros mensuels). Cette dualité rappelle la structure en deux classes de la population française souvent invoquée par les analystes en sciences sociales : d'un côté, le «secteur protégé » des ménages n'ayant pas (encore ?) subi la crise, auxquels viennent se joindre les ménages fortunés ayant les capacités de réagir à la conjoncture ; et de l'autre, le «secteur exposé » regroupant les victimes de la crise. 
Par ailleurs, un ménage sur deux pense que la crise va avoir des conséquences négatives "très importantes" ou "assez importantes" sur leur patrimoine et sur leurs revenus, deux sur trois anticipent une baisse de pouvoir d'achat, et un tiers craint pour son emploi. Ces perspectives négatives se retrouvent également sur les dépenses de consommation : près des deux tiers des enquêtés pensent qu'en raison de la crise il est "très ou assez" probable qu'ils devront baisser leur niveau de vie dans l'année qui vient.

La segmentation "secteur exposé/secteur protégé" ressort également des réponses à la question: «Diriez-vous que depuis la crise financière, vous êtes devenus plus prudent, moins prudent, ou vous n'avez pas changé ». La moitié des ménages n'ont pas changé, une toute petite minorité (1\%) est prête à prendre plus de risque, et les autres (48\%), qui se recrutent principalement parmi les classes les plus défavorisées, se déclarent plus précautionneux. Par ailleurs, $45 \%$ des ménages, appartenant en majorité à ces milieux modestes, se déclarent également plus prévoyants, alors que seulement $1 \%$ se définit comme plus myope (la moitié ne voit pas de changement). Enfin, dans un autre registre, un ménage sur cinq se révèle plus solidaire envers autrui alors qu'inversement, un sur dix se recroqueville sur lui-même (plus individualiste), les deux tiers restant n'ayant pas changé.

Toujours dans la même enquête de juin 2009, les ménages étaient interrogés sur leurs intentions futures en matière d'investissements financiers ou immobiliers. Pour les ménages qui mettent de l'argent de côté, plus de la moitié de la population n'a pas l'intention de modifier son comportement d'épargne alors que parmi les réactifs, la majorité (un tiers) déclare vouloir diminuer son épargne pour pallier la stagnation ou la perte de pouvoir d'achat (un sur 10 déclare vouloir épargner davantage). Parmi ceux qui avaient un projet immobilier (36\% de l'échantillon), quatre ménages sur dix ont décidé de retarder leur décision, $13 \%$ de l'anticiper, le reste n'ayant pas modifié le plan initial.

Parallèlement, on observe une intention majoritaire d'investir dans des actifs plus sûrs. Si l'on compare, pour les détenteurs d'un produit financier donné, le pourcentage de ceux désirant investir davantage d'argent ou autant d'argent dans ce produit au pourcentage de ceux qui ont au contraire l'intention de moins investir ou aussi bien d'arrêter de placer dans ce produit, on voit sur le graphique 1 que la différence est largement positive pour les liquidités et les quasi-liquidités, plus que favorable (autour de $25 \%$ ) pour les assurances et les produits d'épargne logement, encore positif (+20\%) pour les livrets d'épargne et les produits retraite. Par contre, le marché boursier, à cause de la chute des cours, se voit déserté : $-15 \%$ pour les obligations, $-18 \%$ pour les Fonds communs ou les Sicav, et $-20 \%$ pour les actions ${ }^{4}$. De plus, parmi les non possesseurs du produit financier considéré, $10 \%$

\footnotetext{
${ }^{4}$ C'est ce qui ressort aussi de l'enquête lorsque l'on demande directement aux épargnants s'ils ont l'intention de se tourner vers des placements moins risqués dans les mois à venir : ils sont près de deux tiers à déclarer juger "très probable" cette possibilité.
} 
projettent de souscrire une assurance vie, et 6 à $7 \%$ d'investir en épargne retraite, sur un livret d'épargne ou un plan épargne logement; mais moins de $1 \%$ désirent entrer sur le marché boursier (actions, obligations ou fonds communs de placement). Le graphique 2a confirme cette désaffection pour les placements risqués. Quand on interroge plus précisément les ménages sur leurs stratégies d'investissement financier, ils sont de plus en plus nombreux entre mai 2007 et juin 2009 à penser qu'on devrait placer toutes ses économies sur des placements sûrs $(64,4 \%$ en 2009 contre $56,8 \%$ en 2007).

Toutes ces évolutions moyennes concernant les réactions des ménages dans ce contexte de crise doivent être nuancées par des analyses plus fines qui tiennent compte de certaines hétérogénéités individuelles. Ainsi, ces réactions ne sont pas homogènes par rapport à l'échelle des revenus ou des patrimoines, entre les secteurs "exposés" et les "secteurs protégés", chez les riches actionnaires ou chez les travailleurs précaires. Pour de nombreux épargnants, les conséquences de la crise semblent avoir été limitées, voire négligeables, alors que pour d'autres, moins privilégiés, le dilemme entre un désir de précaution accrue d'un côté et la volonté de maintenir son niveau de vie de l'autre s'est posé avec acuité. Ainsi, les ménages fortunés, même s'ils déclarent des pertes patrimoniales importantes, sont beaucoup moins nombreux à prévoir une baisse de leur consommation dans l'année qui vient. Néanmoins, un constat ressort clairement : les ménages français, face à la crise, ont adopté en moyenne un comportement plus prudent, désirent épargner davantage dans des placements plus sûrs et à plus long terme, et veulent limiter leurs investissements risqués.

Simple adaptation au nouvel environnement économique ou changement de préférences?

Comment interpréter le surcroît de prudence des épargnants français mis en évidence précédemment? Provient-il d'une modification des préférences (hausse de l'aversion au risque et/ou du degré de prévoyance), d'une révision des anticipations boursières en matière de rendement ou de volatilité des actifs, ou d'une adaptation au nouvel environnement macroéconomique (hausse du risque de chômage, évolutions futures des revenus salariaux plus incertaines, etc.)?

Les deux vagues de l'enquête Tns-Sofres réalisées en mai 2007 et en juin 2009, soit à des dates qui entourent bien la faillite de Lehman Brothers à l'automne 2008, nous offrent la possibilité d'étudier parallèlement l'évolution de toutes ces composantes du comportement de l'épargnant français pendant la crise financière et économique - et ce notamment pour près de 2250 ménages présents dans les deux vagues. C'est en particulier le cas pour les préférences individuelles à l'égard du risque et du temps, pour lesquelles on dispose de plusieurs mesures directes qui peuvent être comparées entre elles. 
Ces données nous montrent déjà que la crise a eu un impact sur les anticipations des ménages relatives aux marchés boursiers et au marché de l'emploi. Le graphique $2 b$ montre que les rendements (moyens) anticipés sur les actions ont diminué de 2 points (5,5\% contre $3,2 \%$ ) entre 2007 et 2009 et le graphique 2 c révèle un constat similaire sur les anticipations concernant l'évolution de ses propres revenus futurs (de 3,2\% à 1,7\%). Ce pessimisme concernant les perspectives de revenus est d'ailleurs confirmé par les anticipations des ménages concernant leur probabilité de chômage futur qui est plus élevée (de 4\%) en 2009 qu'en 2007. D'un côté, des perspectives de rendements boursiers revus à la baisse, de l'autre des prétentions salariales plus modestes; tout concourt à détériorer la perception de la situation financière par les ménages, qui s'estiment également plus exposés aux risques. Ces évolutions pourraient donc expliquer, au moins en partie, le fait que les ménages aient adopté des comportements plus prudents. Mais qu'en est-il de l'évolution des préférences ?

Les travaux empiriques sur l'épargne demeurent en général assez discrets sur l'évolution temporelle des préférences des agents. Sahm (2008), à partir de choix hypothétiques de loteries ( $c f$. infra), conclut à une relative stabilité de l'aversion relative au risque; pour un même individu, celle-ci augmenterait cependant avec l'âge, mais aussi avec une détérioration de l'environnement macro-économique ${ }^{5}$. Malmendier et Nagel (2010) trouvent, à l'image des «bébés de la Grande Dépression », que les générations qui ont connu des « histoires macroéconomiques » marquées par des marchés boursiers déprimés sont moins enclins à investir dans des actifs risqués, boursicotent moins et se montrent pessimistes dans leurs anticipations des rendements futurs.

\section{IV- MESURER DIRECTEMENT LES PREFERENCES : DIVERSES METHODES}

Chaque questionnaire (de 1998 à 2009) inclut une série de mesures des préférences obtenues à l'aide de différentes méthodes. Les unes, dites «conventionnelles », sont proposées dans la littérature. Les autres proviennent d'une approche originale, fondée sur une procédure de scoring, que nous avons élaborée dès le questionnaire Insee de 1998 et qui a subi des mises au point et améliorations successives depuis. Quoi qu'il en soit, nous avons pris soin de proposer les mêmes mesures, conventionnelles ou scores, aux deux dernières vagues de 2007 et 2009, afin d'obtenir des comparaisons non biaisées des préférences, notamment pour les ménages suivis entre les deux dates.

\footnotetext{
${ }^{5}$ Les autres facteurs démographiques et socioéconomiques expliqueraient la forte hétérogénéité de l'aversion au risque entre individus, mais pas ses variations au cours du temps pour un même individu.
} 


\section{Mesures conventionnelles des préférences}

Nous ne donnerons ici que quelques exemples ${ }^{6}$. En ce qui concerne les attitudes face au risque, la mesure la plus connue et la plus utilisée et celle de l'aversion relative vis-à-vis du risque initiée par Barsky et al. (1997). Elle est fondée sur des choix hypothétiques de loteries concernant le revenu permanent de l'individu. Ce dernier se voit offrir différents contrats de travail à la place de celui actuel, générant un revenu de cycle de vie $R$ : par exemple, un contrat où il a une probabilité $1 / 2$ de gagner $2 R$ et une probabilité $1 / 2$ de gagner seulement $2 / 3 R$. La méthode permet finalement de classer les individus en quatre catégories, des plus « risquophiles » aux plus tolérants au risque. Elle est notamment utilisée sur données de panel par Sahm (2008).

D'autres mesures de l'attitude à l'égard du risque proposent à l'enquêté de se positionner lui-même sur des échelles graduées de 0 à 10 , selon la perception qu'il a de son attitude à l'égard du risque : 0 correspond "très prudent" et 10 à "aventureux" (les deux items sont décrits plus en détail !). Ces échelles auto-évaluées variant de 0 à 10 peuvent être posées "en général" ou concerner des domaines spécifiques de la vie : la santé, le sport et les loisirs, les domaines professionnel ou financier, etc. (Dohmen et al., 2009).

Les mesures de la préférence temporelle pour le présent, plus précisément du taux de dépréciation du futur sont plus fragiles comme le montre le survey peu flatteur de Frederick et al. (2002). On dispose néanmoins d'une échelle auto-évaluée de la préférence pour le présent entre 0 ("vit au jour le jour") et 10 ("préoccupé par l'avenir").

Compte tenu des imperfections de ces indicateurs pour appréhender les comportements de l'épargnant, nous avons initié une méthode plus hétérodoxe de mesure des préférences. Elle consiste à élaborer des scores pour « profiler » les individus suivant leur appétence pour le risque et leur façon d'appréhender le futur. Cette méthode testée à partir des données des enquêtes « Patrimoine 1998 » de l'Insee puis de celles de l'enquête Tns-Sofres de 2002 a été reconduite à partir des informations des deux vagues de l'enquête Tns-Sofres de 2007 et 2009.

Une méthode alternative originale : l'élaboration de "scores" synthétiques et ordinaux

Ces scores synthétiques et ordinaux sont calculés à partir de tout un ensemble de questions concernant différents domaines de la vie, comme la consommation, les loisirs, les placements, le travail, la famille, la santé, la retraite... Au total, ces scores sont construits à partir d'une centaines de questions, le score de risque retenant par exemple une soixantaine d'entre elles (voir Arrondel et Masson, 2007 et 2008).

\footnotetext{
${ }^{6}$ Pour une recension détaillée de ces mesures conventionnelles des préférences à l'égard du risque et du temps, qui incluent également des mesures sur données expérimentales, voir Arrondel et Masson (2010).
} 
A travers ces nombreuses questions de différente nature (de comportement, d'opinion ou d'intention, de réactions à des loteries ou à des scénarios fictifs...) et qui balaient un large éventail des domaines de l'existence, l'objectif est de construire pour chaque enquêté des indicateurs relatifs cohérents - des "scores" qualitatifs - qui évaluent par exemple son attitude générale à l'égard du risque et de l'incertain (plutôt que précisément son degré d'aversion au risque ou de prudence...).

Avant d'expliciter et d'illustrer la méthode, abordons le problème du nombre de paramètres de préférence à introduire. Le choix en cette matière dépend déjà de considérations théoriques : un modèle de cycle de vie élargit conduit ainsi à distinguer trois grandes motivations pour l'accumulation d'un patrimoine: la précaution, liée à la «prudence » et donc à l'aversion au risque ; le lissage intertemporel des consommations et l'épargne pour les vieux jours, fonctions du degré de «prévoyance»; et le patrimoine destiné à la transmission, qui dépend du degré d'altruisme familial ; en outre, les choix de portefeuille dépendent surtout, dans ce cadre, de l'aversion pour le risque. On sait toutefois que ce modèle basique décrit mal les comportements des épargnants : en particulier, d'autres paramètres de préférence apparaissent nécessaires pour rendre compte de leur rationalité « limitée ». La théorie mais aussi les données nous ont ainsi conduit à considérer au départ quatre paramètres de préférence ${ }^{7}$ :

- $\gamma$ représente les attitudes face au risque (aversion, prudence, etc.);

- $\delta$ représente la préférence de long terme pour le présent, soit le taux de dépréciation du futur sur le cycle de vie ;

- $\beta$ représente l'impatience à court terme, qui témoigne en général d'une incohérence temporelle des choix (rationalité limitée) liée à une faiblesse de la volonté ou un déficit d'imagination - voir Laibson (1997) ;

- $\theta$ mesure le degré d'altruisme familial, soit le poids accordé au bien-être de ses enfants par rapport à sa propre consommation.

Pour chaque (type de) préférence que l'on cherche à mesurer - attitude à l'égard du risque $(\gamma)$, préférence pour le présent $(\delta)$, impatience à court terme $(\beta)$, degré d'altruisme familial $(\theta)$-, nous avons retenu a priori un certain nombre de questions ; certaines d'entre elles, de nature polysémique, ont été affectées à deux indicateurs à la fois, notamment $\gamma$ et $\delta$ (le futur est à la fois incertain et éloigné du présent).

Donnons quelques exemples de questions. En matière d'attitude à l'égard du risque, on trouve aussi bien des cas anecdotiques, du genre : "Prenez-vous un parapluie lorsque la météo est incertaine", ou "Garez-vous votre véhicule en état d'infraction", que des choix de loteries, des pratiques de consommation : «Vous arrive-t-il d'aller au spectacle un peu au

\footnotetext{
${ }^{7}$ Pour la justification de ce choix, voir Arrondel et Masson (2007).
} 
hasard au risque d'être parfois déçu »?" ou encore des opinions : "Êtes-vous d'accord avec l'affirmation que le «mariage est une assurance » ?" ou bien : "Êtes-vous sensible aux débats de santé contemporains (sida, sang contaminé...)".

Une question de référence pour identifier $\delta$ est : "Suite à une charge de travail inopinée, votre employeur vous demande de reporter d'un an une semaine de vacances quitte à vous attribuer $x$ jours supplémentaires de congé...". Quant aux items "Pensez-vous que cela vaut la peine, pour gagner quelques années de vie, de se priver de ce qui constitue pour soi les plaisirs de l'existence," et "Pour éviter des problèmes de santé, surveillez-vous votre poids ou votre alimentation, faites-vous du sport...", ils ont été affectées à la fois à $\gamma$ et à $\delta$.

L'interprétation des réponses apportées à ce genre de questions de la vie de tous les jours pose problème en raison notamment des effets de contexte et de facteurs non pertinents (un individu amoureux du risque peut, par civisme, ne jamais se garer en zone interdite). L'idée sous-jacente est que seule la moyenne des réponses aurait un sens, pourvu qu'elle soit suffisamment représentative. La méthode statistique consiste alors à coder chaque question nous l'avons fait en général en trois modalités (ainsi pour $\delta:-1$ : imprévoyant ; 0 position moyenne $;+1$ : prévoyant) -, puis à sommer les "notes" obtenues par l'individu ; son score est enfin la somme des notes réduite aux seuls items qui se sont révélés, ex post, former un tout statistiquement cohérent (selon le critère de l'alpha de Cronbach $^{8}$ qui élimine les questions les moins contributives). Les scores sont donc des mesures synthétiques, qualitatives et ordinales, supposés représentatifs des réponses fournies par l'enquêté à un ensemble de questions diverses ( $c f$. Arrondel et al., 2004).

S'agissant du nombre de scores à introduire, les données ont donc le dernier mot: l'analyse statistique peut ainsi révéler que plusieurs scores de risque, présentant un degré de cohérence interne suffisant, doivent être introduits, en distinguant par exemple les réactions aux petits risques et aux risques vitaux, des scores par domaine de la vie, etc. Or sur les quatre enquêtes, nous avons pu montrer qu'un seul score suffisait pour chaque préférence $\gamma$, $\delta, \beta$, et $\theta$. Ce résultat révèle déjà la robustesse de la méthode utilisée. ${ }^{9}$

\section{La supériorité des scores sur les autres mesures de préférence}

On montre (Arrondel et Masson, 2010) que les préférences mesurées par les scores synthétiques ont de meilleures performances que les mesures conventionnelles, loteries et échelles en particulier.

\footnotetext{
${ }^{8}$ Pour plus de détails sur la méthode de construction des scores et leur propriétés, on peut se référer à Arrondel et Masson (2010).

${ }^{9}$ La construction des scores a légèrement évolué d'une enquête à l'autre. Mais nous avons pris soin de poser les mêmes questions dans les deux vagues Tns-Sofres de 2007 et 2009 afin de construire des scores de préférence les plus comparables possible et pouvoir étudier les changements éventuels des différents paramètres de préférences, en particulier pour les 250 ménages qui ont répondu aux deux vagues de l'enquête.
} 
Les histogrammes des scores présentent ainsi des propriétés de dispersion et de régularité, qui plus est comparables d'une enquête à l'autre (voir Graphique 3), que l'on ne retrouve pas pour les autres mesures : la mesure fondée sur des loteries de Barsky et al. (1997) n'a que quatre modalités, les distributions obtenues pour les échelles ont un mode fluctuant d'une enquête à l'autre, etc.

Par ailleurs, les scores mesurés à partir des informations des différentes enquêtes apparaissent cohérents d'une date à une autre: les classements des questions les plus pertinentes pour expliquer les scores présentent la même hiérarchie; et les déterminants individuels des scores sont les mêmes d'une enquête à l'autre. Ainsi, lorsque l'on veut savoir « qui est quoi en matière de préférence ? », les résultats, qui vont en général dans le sens attendu, coïncident entre les quatre enquêtes : les hommes sont plus tolérants au risque que les femmes, et les jeunes que leurs aînés; on voit à plus long terme (faible préférence temporelle) lorsqu'on est âgé, diplômé, en couple et que l'on a des enfants (et la prévoyance semble également se transmettre par la mère du répondant); on est plus altruiste si l'on est diplômé ; mais les plus impatients à court terme n'ont pas de caractéristiques particulières.

Les effets quantitatifs des scores sur les patrimoines et les choix de portefeuille sont en outre plus significatifs et comparables d'un enquête à l'autre. Et ils ne souffrent pas des même biais d'endogénéité que les autres mesures : en effet, par construction, les scores s'apparentent en partie à une collection d'instruments naturels : par exemple, en matière de risque, "prendre ses précautions si la météo est incertaine » constitue une question très corrélée avec la tolérance au risque des individus, mais ne permet pas évidemment d'expliquer leurs comportements patrimoniaux !

Les corrélations entre les scores différents scores de préférence sont également très proches d'une enquête à l'autre : par exemple une moindre tolérance au risque va avec une plus grande prévoyance (corrélation autour de 0,40 ).

Enfin, pour les individus panélisés ayant répondu en 2007 et 2009, les scores, pour un même paramètre, sont fortement corrélés d'une enquête à l'autre : 0,65 pour le temps, 0,75 pour le risque ; pour les autres mesures de l'attitude à l'égard du risque, notamment, les corrélations temporelles sont beaucoup plus faibles, autour de 0,30 (voir Tableau 1).

\section{V- LES EFFETS DE LA CRISE SUR LES PREFERENCES DE L'EPARGNANT FRANÇAIS}

La question centrale de cet article concerne l'interprétation du surcroît de prudence des épargnants Français pendant la crise, question qui remet en lumière une hypothèse fondamentale de l'analyse économique à savoir la stabilité temporelle des préférences. Pour cela, il s'est agi de «profiter » de la crise, non seulement pour comparer les comportements d'épargne, d'accumulation et de composition du patrimoine des ménages avant et après ce 
choc conjoncturel, mais aussi de mesurer son impact sur les préférences de l'épargnant. Cette analyse a pu être menée à partir de l'enquête Tns-Sofres sur le sous-échantillon des 2234 ménages ayant répondu aux deux vagues du questionnaire en Mai 2007 et en Juin 2009.

Les analyses précédentes sur ces données ont montré que les épargnants français se voyaient plus prudents ou averses au risque dans leurs comportements : davantage d'épargne de précaution, révision de la consommation à la baisse, désir de modérer sa prise de risque dans les portefeuilles. La questions qui se pose est de savoir si ces changements doivent être imputés à des modifications des préférences elles-mêmes, en particulier de l'aversion au risque, ou bien si les ménages perçoivent différemment leur environnement économique, qu'il s'agisse de leur situation vis-à-vis du marché du travail, de leurs anticipations relatives au rendement et à la volatilité des actifs boursiers, ou de l'ampleur des risques qu'ils subissent en dehors des marchés financiers ?

Les informations quant aux anticipations des ménages concernant le marché boursier (rendements espérés moindres) ou le marché du travail (revenus anticipés revus à la baisse, risque de chômage accru) allait bien dans le sens d'une plus grande prudence des ménages. Mais les préférences de l'épargnant prennent-elles le même chemin ?

\section{Des mesures traditionnelles montrant une moindre tolérance au risque}

Sur les échelles de la tolérance au risque, auto-évaluées de 0 à 10 , les ménages se positionnent en moyenne de manière plus prudente en 2009 qu'en 2007 : le graphique 4 montre, en effet, que la moyenne de l'échelle générale augmente de 4,3 à 4,7; il en va de même dans les différents domaines envisagés, en particulier quant aux placements financiers.

De manière analogue, la loterie de Barsky et al. (1997) révèle une augmentation de l'aversion relative pour le risque avant et après la crise financière (tableau 2) : en particulier, le groupe des moins tolérants vis-à-vis du risque augmente de près de 10 points $(47 \%$ vs. $56 \%)$.

Les autres mesures subjectives du type de celle de Kapteyn et Teppa (2009) ou de Iezzi (2008), qui consistent à interroger directement les ménages sur leurs intentions en matière d'investissement aboutissent à la même conclusion (voir graphique 2a) : les ménages seraient devenus plus prudents, comme en témoigne le nombre de plus en plus important de réponses en faveur d'une absence totale de prise de risque dans le portefeuille financier $(64,4 \% v s$. $56,8 \%)$.

Le constat est clair: toutes les mesures habituellement utilisées pour mesurer les préférences des individus à l'égard du risque semblent avoir été affectées dans le même sens 
pendant la crise : les ménages Français sont moins tolérants au risque, en particulier dans le domaine de l'épargne et des choix de portefeuille.

Si l'on s'intéresse maintenant aux préférences à l'égard du temps, les échelles d'autoévaluation indiquent que les ménages seraient également devenus un peu plus prévoyants sur le long terme et aussi un peu moins impatients - plus posés - sur le court terme (voir graphique 4).

Des scores de préférences à l'égard du risque et du long terme distribués de manière identique

Comparons maintenant les histogrammes des scores de risque construits à partir des mêmes questions pour la sous-population des ménages ayant été interviewés avant et après la chute de Lehman-Brothers (graphique 5a). On constate que les deux histogrammes se superposent presque parfaitement, avec la même moyenne aux deux dates. Les scores nous indiquent donc que les épargnants n'ont pas "changé" : ils sont en moyenne tout aussi tolérants au risque qu'avant la crise, ni plus, ni moins. D'ailleurs, l'histogramme des changements individuels des scores entre les deux dates (graphique 5b) révèle une distribution purement aléatoire (de forme gaussienne), avec autant de ménages plus tolérants au risque que de ménages moins tolérants.

Nous avons procédé au même exercice sur les scores de préférence temporelle. Le graphique 6a révèle la même stabilité d'ensemble : les deux histogrammes coïncident et les caractéristiques de la distribution (moyenne et médiane) sont les mêmes. Encore une fois, la distribution des variations individuelles suit une courbe de forme gaussienne, apparemment aléatoire (graphique 6b).

Enfin, on peut s'intéresser aux deux autres paramètres calculés à partir de la même méthode de scoring, à savoir l'impatience à court terme et l'altruisme. La comparaison des distributions aux deux dates montre alors que les ménages seraient devenus plus altruistes envers leurs enfants (repli sur la famille et ses "valeurs" ?) mais aussi moins impatients - ce qui irait dans le même sens que l'échelle d'impatience (graphiques 7a et 7b).

\section{Des changements de préférence individuelle difficiles à expliquer}

Pour pouvoir conclure définitivement à la stabilité des préférences des épargnants, ces résultats nécessitent cependant une analyse plus poussée que cette simple étude descriptive des tendances moyennes.

Une première étape consiste ainsi, à l'instar de Sahm (2008), à étudier les variations "transitoires" individuelles des préférences sur les deux ans afin de savoir s'il s'agit d'un phénomène purement aléatoire ou bien si ces variations peuvent être expliquées par des caractéristiques individuelles ou des données macroéconomiques. Les premières études 
économétriques ne permettent pas de mettre en évidence d'effet significatif des changements des caractéristiques individuelles, pas plus que des changements de perception du contexte économique (le pouvoir explicatif global de la régression est de plus très faible). Seuls les ménages exprimant le sentiment d'être "très concerné par la crise" se révèleraient plus prudents selon notre score.

\section{Des mesures traditionnelles mesurant d'autres phénomène que les préférences}

De nombreux indicateurs semblent montrer que l'épargnant français est devenu plus prudent dans ces comportements patrimoniaux après la crise financière de 2008. Les deux vagues de l'enquête Tns-Sofres nous permettent d'isoler les changements des différents déterminants des comportements d'épargne, d'accumulation et de composition du patrimoine : évolution des revenus, instabilité des préférences, révision des anticipations, plus grande exposition aux risques non financiers.

Les informations recensées dans ces enquêtes montrent tout d'abord que les ménages estiment que leur avenir professionnel est plus sombre (hausse de la probabilité anticipée de chômage, baisse des revenus futurs attendus...) et espèrent des rendements moindres de leurs actifs patrimoniaux. Le constat relatif aux préférences dépend quant à lui de la méthode suivie pour les évaluer.

Les mesures issues de notre méthode des scores montrent que les préférences à l'égard du risque et du temps n'auraient pas changé durant la crise, alors que les autres mesures, loteries et échelles, qui concluent à une diminution de la tolérance au risque des agents et à une prévoyance accrue. $\mathrm{Si}$, comme nous le démontrons, nos mesures présentent de meilleures propriétés que les autres (Arrondel et Masson, 2010), ces conclusions signifieraient que les indicateurs habituellement utilisés dans la littérature mesurent en fait un composé hétérogène de préférences per se et d'autres éléments subjectifs plus volatiles, concernant l'exposition au risque ou encore un certain pessimisme vis-à-vis des marchés.

\section{VI - CONCLUSIONS}

Dans cet article, nous avons cherché principalement à expliquer le désir de prudence accrue exprimé par les ménages après la crise financière de 2008. Es-ce dû à un contexte économique plus incertain, des perspectives d'évolution des revenus revues à la baisse, des anticipations de rendements financiers plus faibles et plus aléatoires, ou enfin à des préférences qui auraient changé lors de la crise?

Pour mesurer ces différents impacts, nous disposions d'une source unique constituée par les deux vagues (avant et après la crise financière de 2008) d'une enquête Tns-Sofres 
destinée à mesurer les préférences et les anticipations des épargnants et parallèlement à quantifier leur niveau de richesse et sa composition.

Les informations issues de cette enquête attestent bien que les ménages sont devenus plus prudents et plus prévoyants dans leurs comportements: hausse de l'épargne de précaution, des perspectives de consommation revues à la baisse, des anticipations de revenus plus pessimistes, des espérances de revenus financiers plus faibles, une volonté d'investir dans des placements plus sûrs... Le constat est moins clair sur l'évolution des préférences. Si les mesures conventionnelles, loteries ou échelles, concluent à une augmentation de l'aversion au risque et du degré de prévoyance, les scores construits à partir de notre méthode consistant à agréger les réponses à un grand nombre de questions, ne semblent pas avoir changé en moyenne.

Plus précisément, les distributions des scores de risque et de préférence temporelle en mai 2007 et en juin 2009 sont restées stables, et les variations individuelles transitoires restent largement aléatoires et ne peuvent être expliquées, ni par les caractéristiques du ménage, ni par les données macroéconomiques. Ces résultats font d'ailleurs écho à ceux de Sahm (2008) qui obtenait pour les Etats-Unis, à partir d'une méthodologie différente de mesure de l'aversion au risque (loterie à la Barsky), une relative stabilité des préférences au cours du temps, certes une période sans choc exogène de l'ampleur de celui de la crise financière présente. Si Sahm montre que l'aversion au risque varie fortement d'un individu à l'autre en fonction de ses caractéristiques, ce paramètre de préférence se montre plutôt stable au cours du temps, et là encore, les variations individuelles sont difficile à expliquer. Cette stabilité des préférences, au moins sur de courtes périodes, même en présence d'un choc macroéconomique comme celui de la crise financière actuelle, justifierait la pratique habituelle d'ignorer les variations potentielles de préférences en général non observées.

La prochaine étape de nos travaux consistera à expliquer, au niveau individuel, les changements des comportements patrimoniaux entre 2007 et 2009 en fonction de la situation objective des ménages et d'autres changements d'ordre plus subjectif, comme les anticipations des revenus futurs, les espérances de rendements des actifs financiers et les variations des préférences.

Par ailleurs, on voit bien dans quelles directions les recherches futures dans ce domaine devront porter. Il s'agira d'une part, de continuer à interroger les mêmes individus sur leurs préférences et leur portefeuille de façon à juger du bien fondé de cette hypothèse de stabilité des préférences à plus long terme. D'autre part, des comparaisons internationales seraient bienvenues pour attester la pertinence de notre méthodologie de mesure des préférences sur d'autre pays que la France. 


\section{Bibliographie}

Arrondel L. et Masson A. (2007), Inégalités patrimoniales et choix individuels. Des goûts et des richesses..., , Economica, Paris,

Arrondel L. et Masson A. (2008), "How to Measure Risk and Time Preferences of Savers ?", mimeo PSE.

Arrondel L. et Masson A. (2010), "French Savers in the Economic Crisis: What has Changed ? Variation of Preferences or Simple Adaptation to a New Environment", mimeo, PSE-Jourdan, Paris.

Arrondel L., A. Masson et D. Verger, "Préférences de l'épargnant et accumulation patrimoniale" (dossier de 5 articles), Economie et Statistique, 2004, n 374-375.

Barsky, R.B., Kimball, M.S., Juster, F.T. et Shapiro, M.D. (1997), "Preference Parameters and Behavioral Heterogeneity: An Experimental Approach in the Health and Retirement Survey", Quarterly Journal of Economics, 112 (2), p. 537-580.

Dohmen T., Falk A., Huffman D., Sunde U., Schupp J. et Wagner G. (2009), “Individual Risk Attitudes: Measurement, Determinants and Behavioral Consequences", ROA-Research Memorandum-2009/6, Research Centre for Education and the Labour Market, Maastricht University.

Frederick S., Loewenstein G et O'Donoghue T. (2002), "Time Discounting and Time Preference: a Critical Review", Journal of Economic Literature, 40, p. 351-401

Iezzi, S. (2008), "Investors' Risk Attitude and Risky Behavior": a Bayesian Approach with Imperfect Information", Bank of Italy Working Paper 692.

Kapteyn A. et Teppa F. (2009), "Subjective Measures of Risk Aversion, Fixed Costs, and Portfolio Choice", DNB Working Paper 216, De Nederlandsche Bank, Amsterdam.

Laibson D. (1997), “Golden Eggs and Hyperbolic Discounting”, Quarterly Journal of Economics, 112, p. 443-477.

Lapègue V. et Mauroux A., 2010, "Crise et dépendances", Note de conjoncture, Insee, 21-34.

Malmendier U. et S. Nagel, «Depression Babies: Do Macroeconomic Experiences Affect Risk-Taking? », May 2010, Quarterly Journal of Economics, forthcoming.

OECD (2010), OECD Factbook 2010: Economic, Environmental and Social Statistics, OECD Publishing.

Sahm C. (2008), "Stability in Risk Preference", Finance and Economics Discussion Series 2007-66. Washington: Board of Governors of the Federal Reserve System. 
Tableau 1 : Correlations des scores de préférence en 2007 et 2009

\begin{tabular}{|c|c|}
\hline & $2007-2009$ \\
\hline Scores de risque préférence temporelle & 0.75 \\
Loteries & 0.66 \\
Echelles de risque & 0.30 \\
Echelles de préférence temporelle & 0.32 \\
\hline Nombre d'observations & 0.54 \\
\hline
\end{tabular}

Source : TNS 2007-2009 (panel)

Tableau 2 : Aversion relative pour le risque en 2007 et 2009 (en \% de la population)

\begin{tabular}{c|c|c|c|c}
\hline \hline \multirow{2}{*}{$\begin{array}{c}\text { Aversion relative pour le } \\
\text { risque }: \gamma\end{array}$} & \multicolumn{2}{|c|}{ Rejet du contrat $A$} & \multicolumn{2}{c}{ Acceptation du contrat $A$} \\
\cline { 2 - 5 } & Rejet du contrat $C$ & $\begin{array}{c}\text { Acceptation du contrat } \\
C\end{array}$ & Rejet du contrat $B$ & Acceptation du contrat $B$ \\
\cline { 2 - 5 } 2007 Panel Sofres & $\mathbf{3 . 7 6 = < \gamma}$ & $\mathbf{2 = < \gamma < \mathbf { 3 . 7 6 }}$ & $\mathbf{1 = < \gamma < \mathbf { 2 }}$ \\
\hline \multirow{2}{*}{2009 Panel Sofres } & 46,8 & 25,5 & 20,0 & 7,7 \\
\hline \hline
\end{tabular}

Le choix est entre un revenu certain et plusieurs contrats. Contrat A : une chance sur deux de doubler son revenu mais une chance sur deux de le voir diminuer d'un tiers. Contrat B : une chance sur deux de doubler son revenu, une chance sur deux de le voir diminuer de moitié. Contrat $\mathrm{C}$ : une chance sur deux de doubler son revenu, une chance sur deux de le voir diminuer d'un cinquième.

Source : TNS 2007-2009 (panel) 
Graphique 1 : "Et dans les mois à venir, pensez-vous placer plus d'argent, moins d'argent ou autant d'argent qu'aujourd'hui dans les placements financiers suivants (ou bien vous n'êtes pas concerné par ces produits) ?"

Plus d'argent ou autant d'argent vs. moins d'argent ou ne plus épargner sur le

$\square$ Plus d'argent $\square$ Autant d'argent $\square$ Moins d'argent $\square$ Arrêter d'épargner sur ce support $\square$ Ne sait pas

$$
\text { produit }
$$

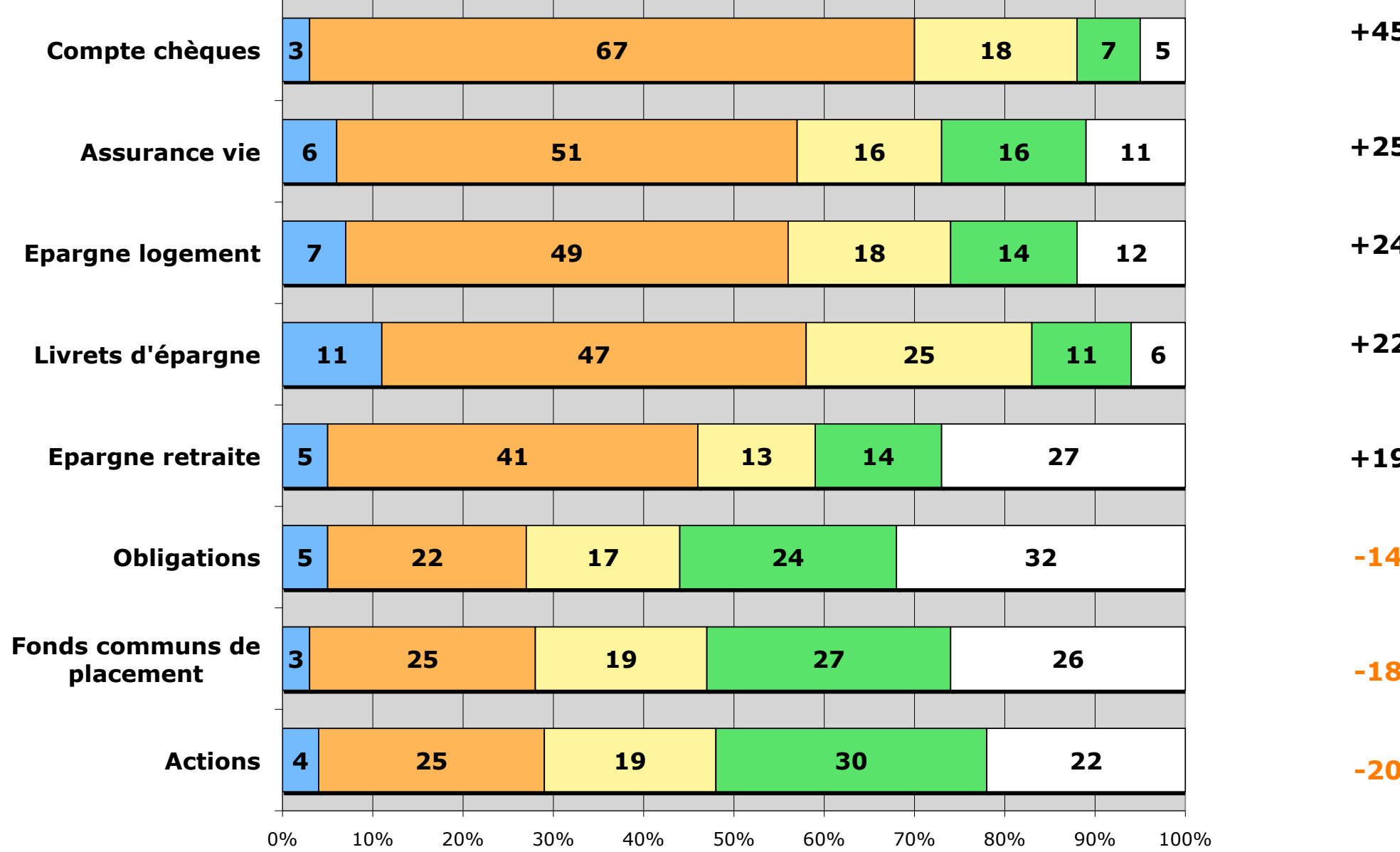

$+45$ 
Graphique 2a : En matière de placements financiers, que préférez-vous (en \%) ? (Panel 2007-2009)

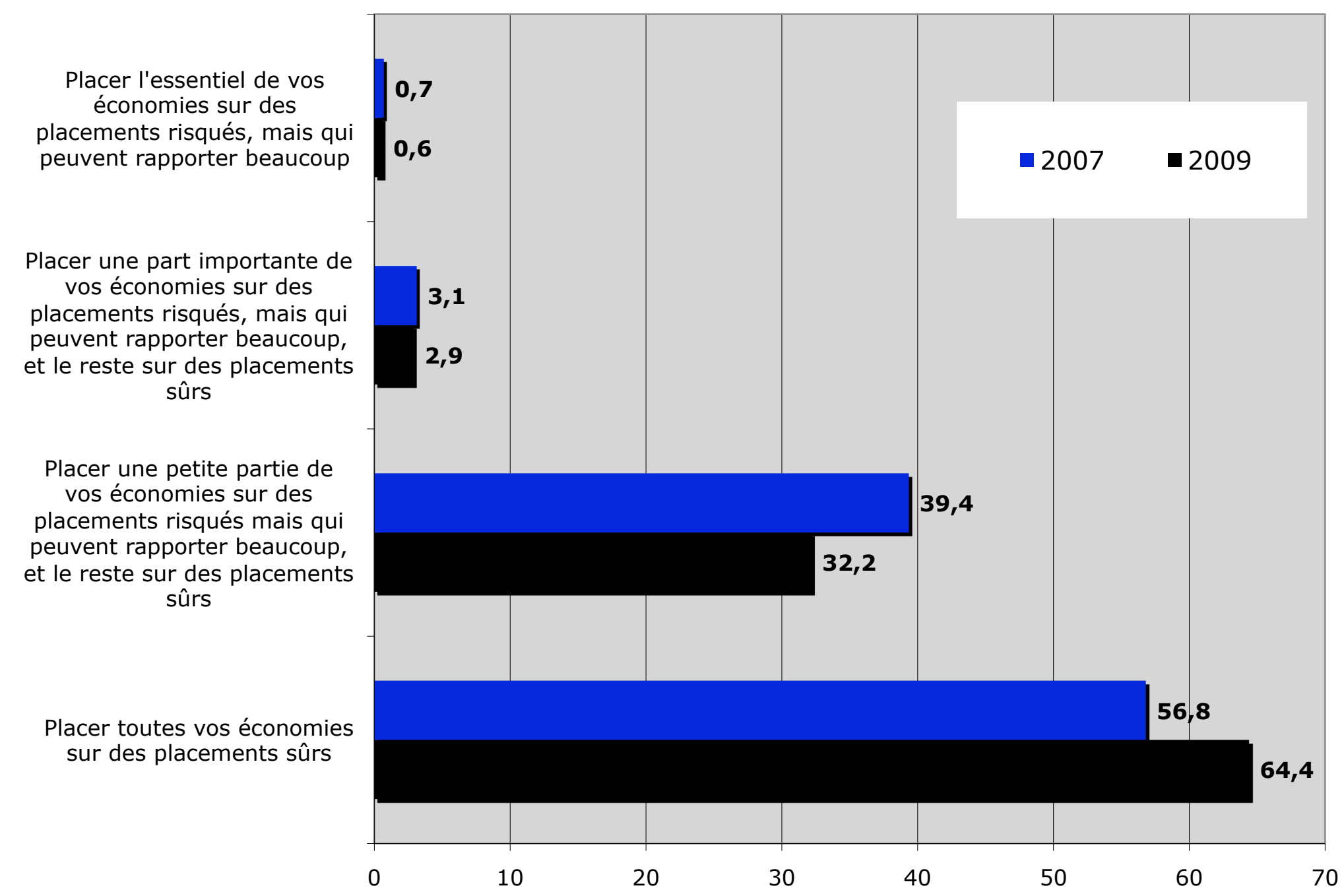

Source : TNS 2007-2009 (panel) 
Graphique 2b : Rendements anticipés (dans les 5 prochaines années) sur le marché boursier en 2007 et 2009

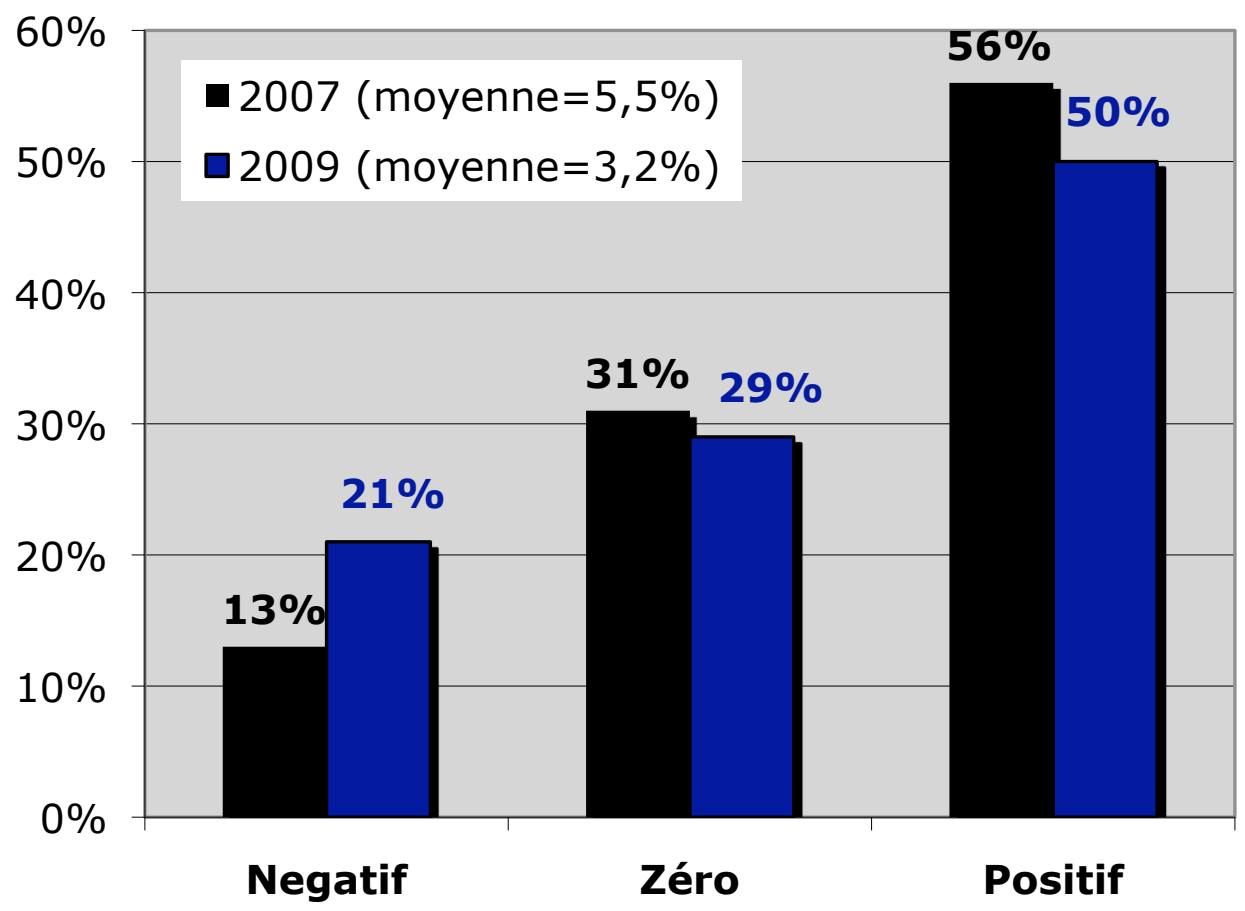

Graphique 2c : Croissance des revenus du travail futurs anticipés (dans les 5 prochaines années) en 2007 et 2009

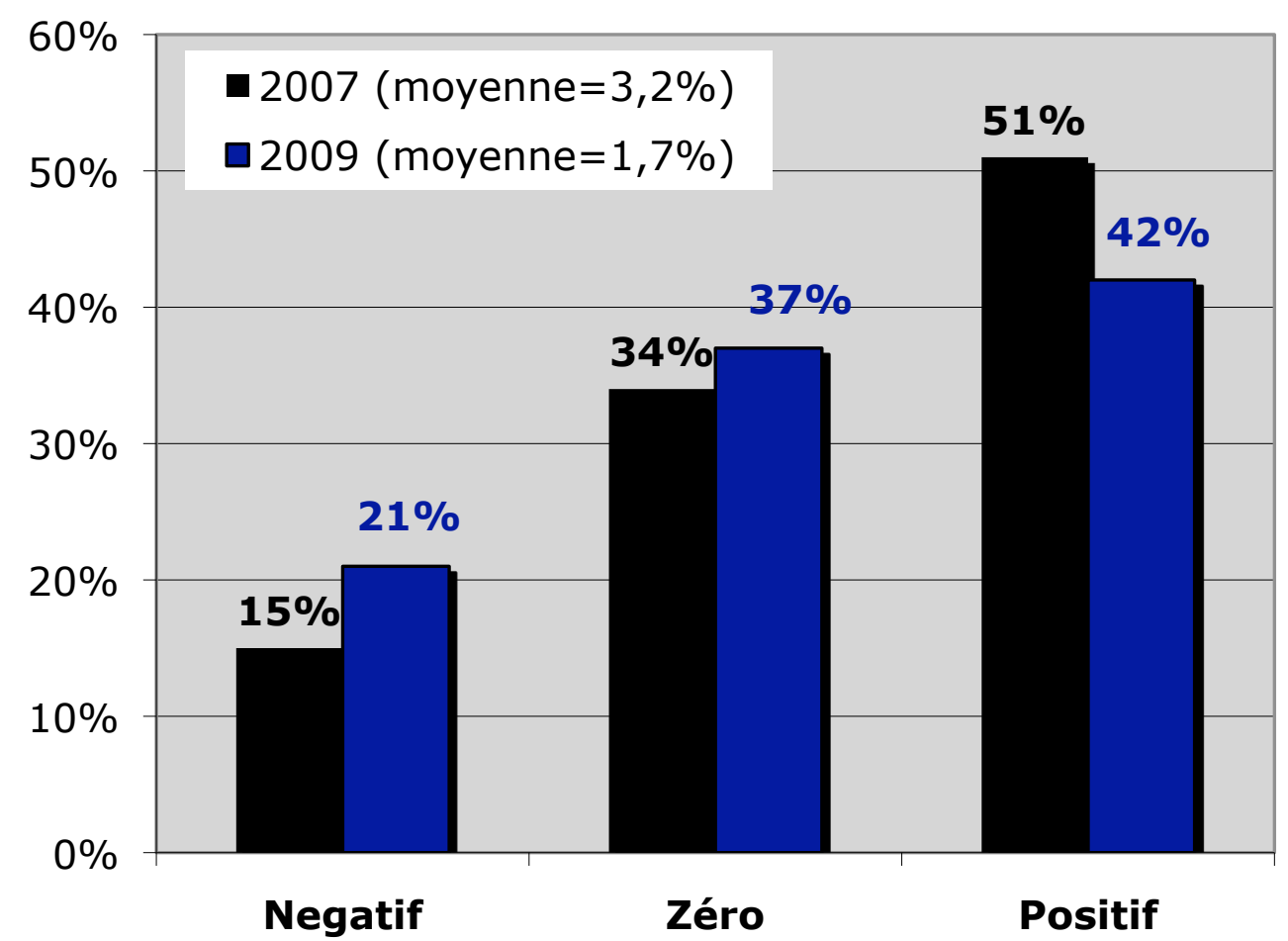

Source : TNS 2007-2009 (panel) 
Graphique 3 : Histogrammes des scores

a) Risque
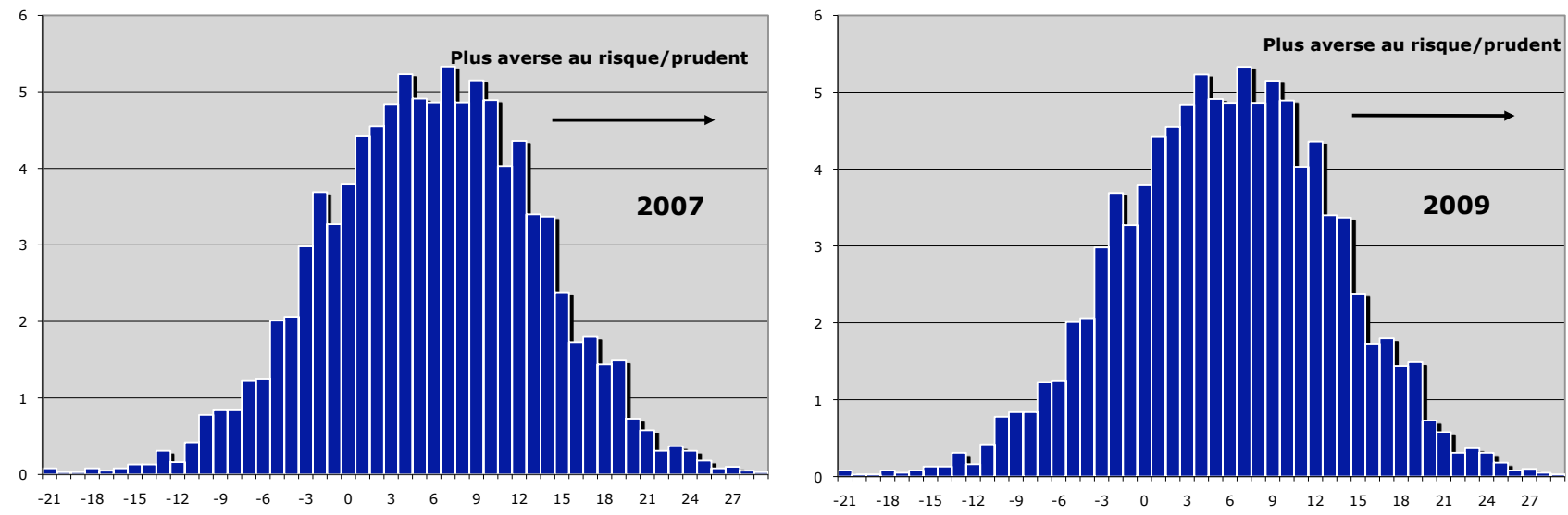

b) Temps
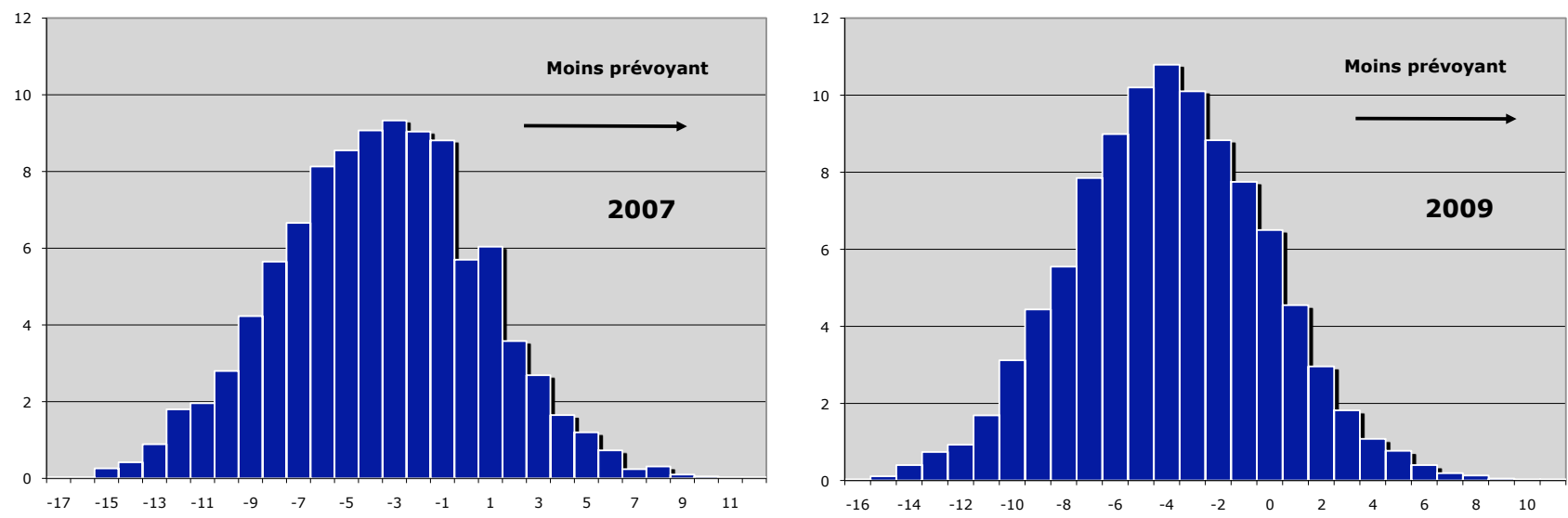

Source : TNS-Sofres 2007 et 2009 
Graphique 4 : Echelles de préférences entre 2007 and 2009

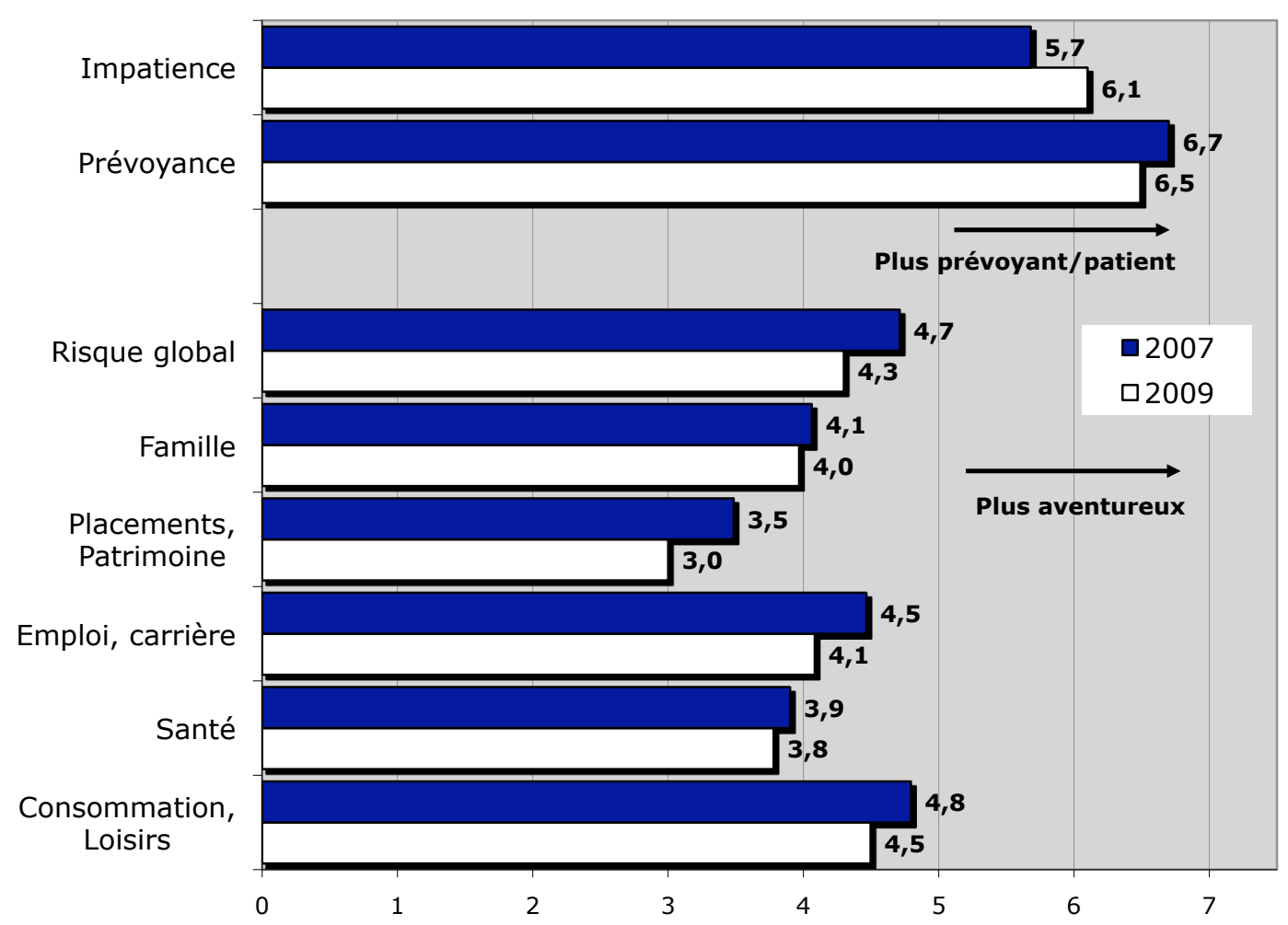

Source : TNS 2007-2009 (panel) 
Graphique 5a : Histogramme du score de risque en 2007 et 2009

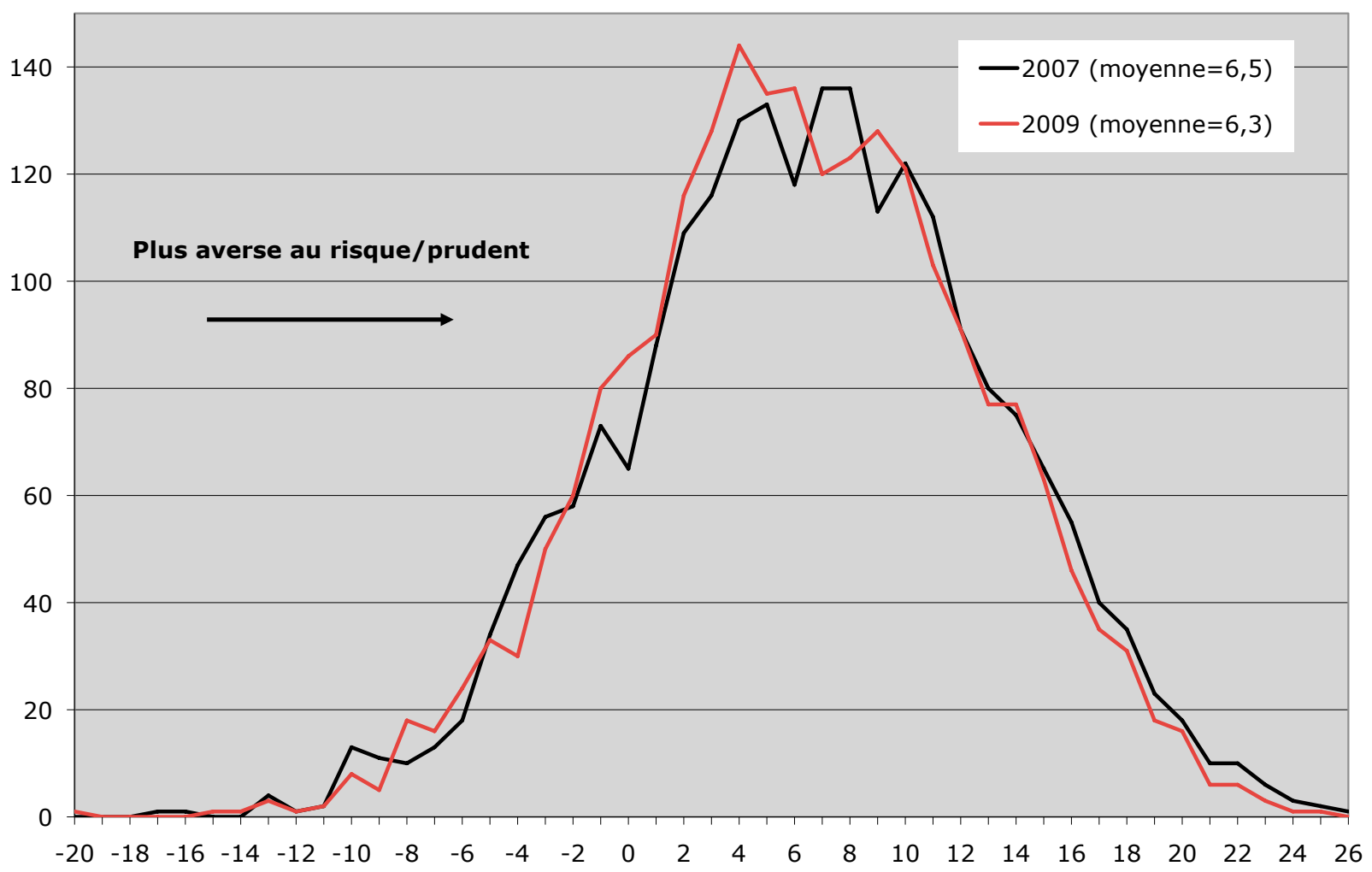

Graphique 5b : Histogramme des changements du score de risque en 2007 et 2009

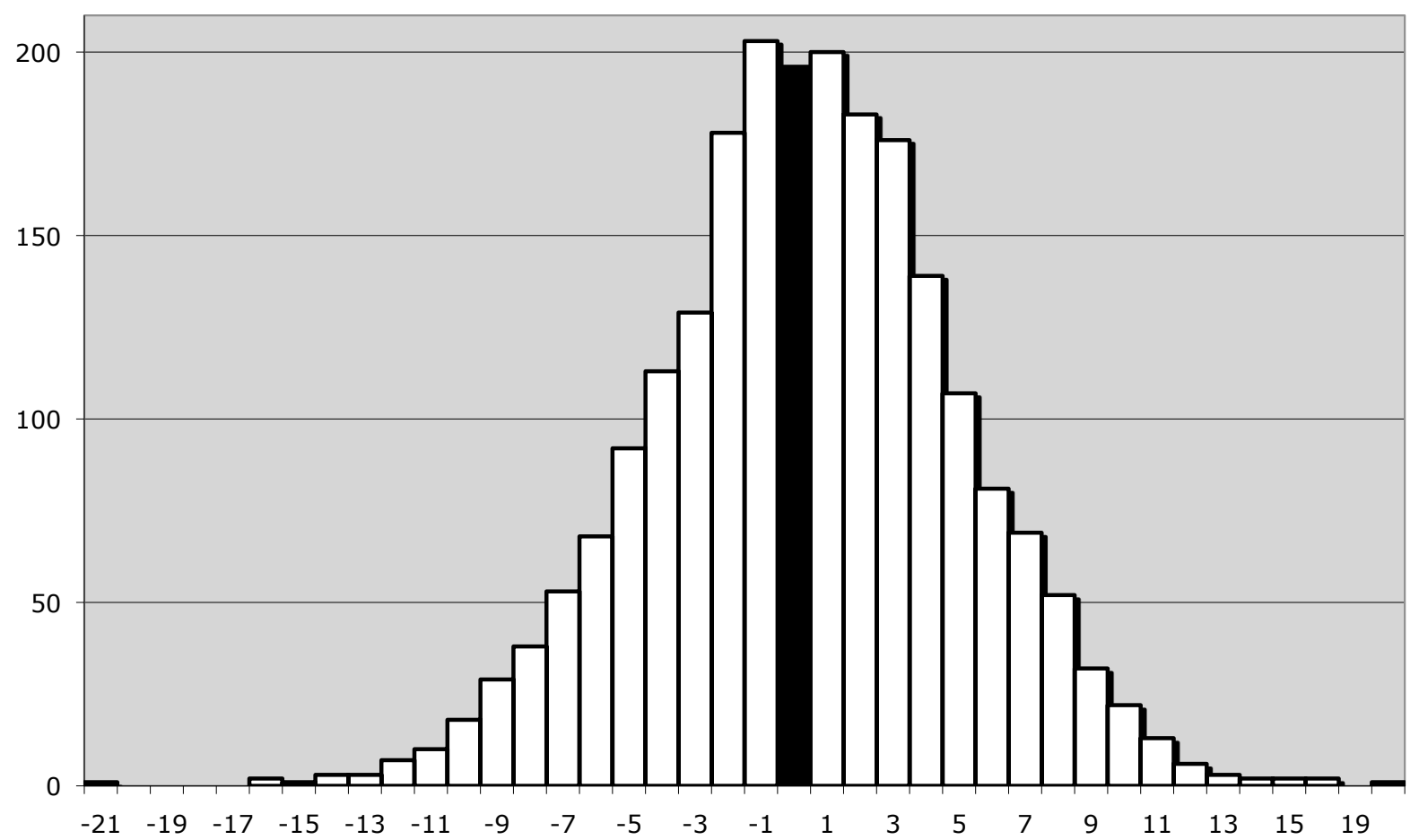

Source : TNS 2007-2009 (panel) 
Graphique 6a : Histogramme du score de préférence temporelle en 2007 et 2009

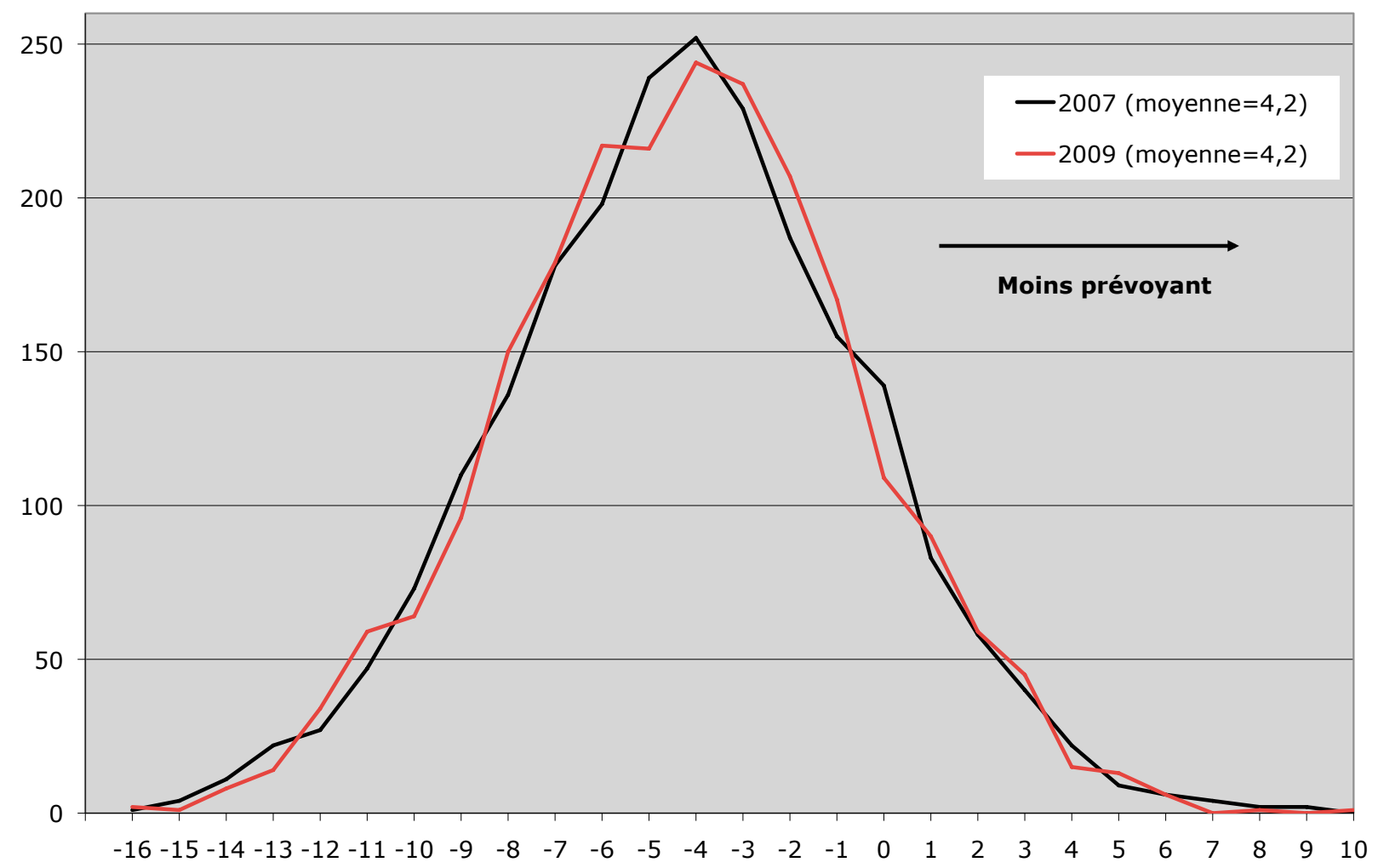

Graphique 6b : Histogramme des changements du score de préférence temporelle en 2007 et 2009

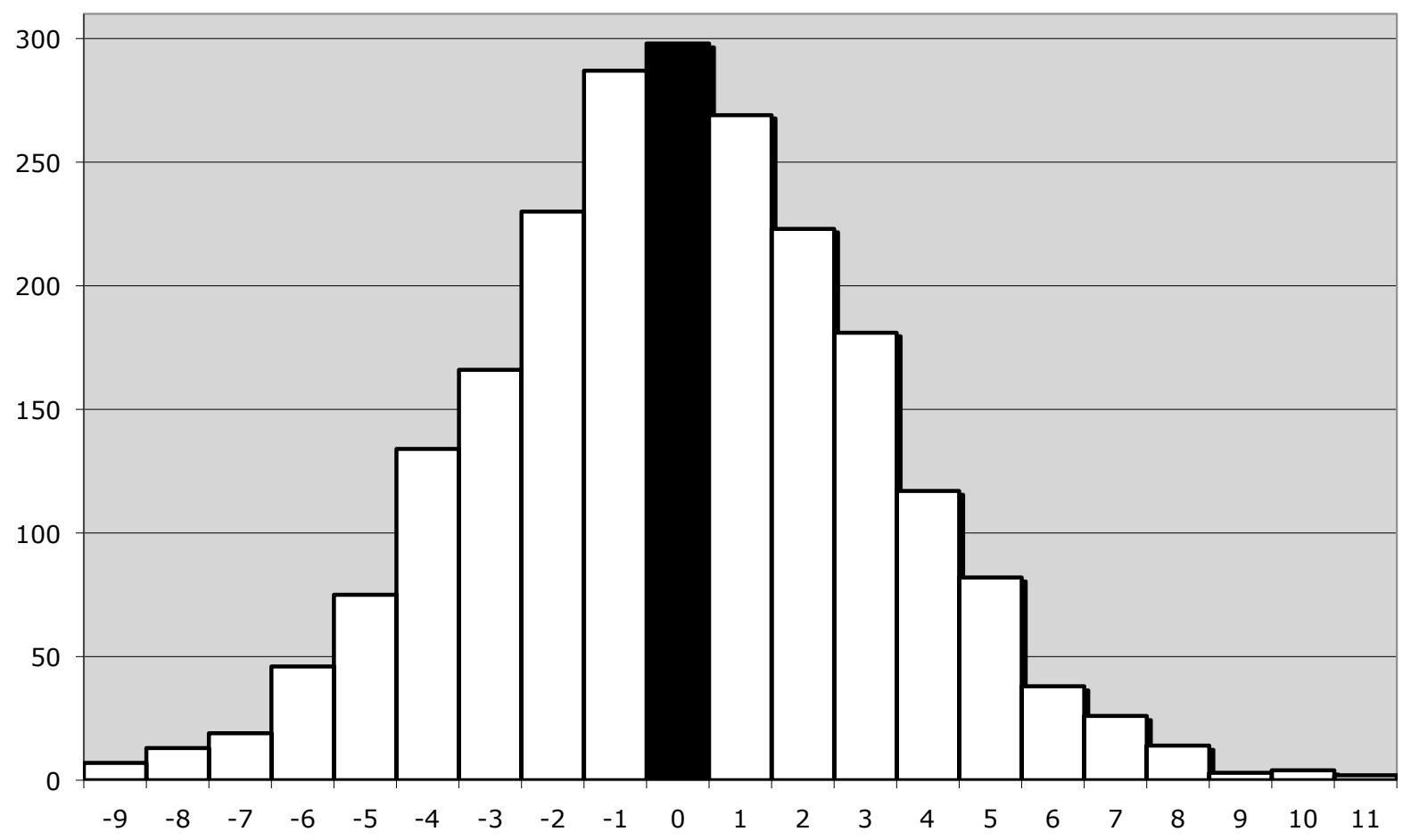


Graphique 7a : Histogramme de l'altruisme familial en 2007 et 2009 (scores)

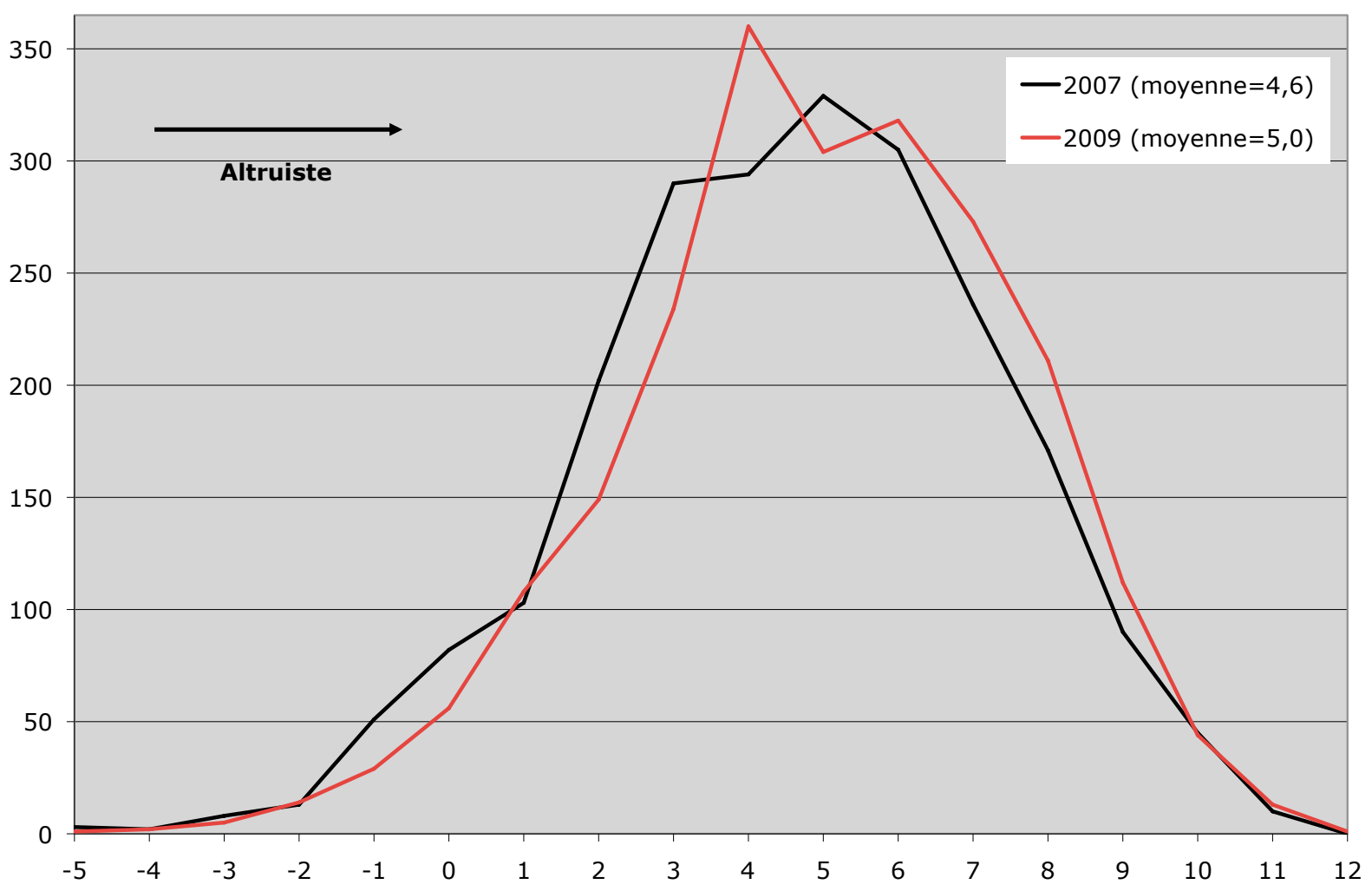

Graphique 7b : Histogramme de l'impatience en 2007 et 2009 (scores)

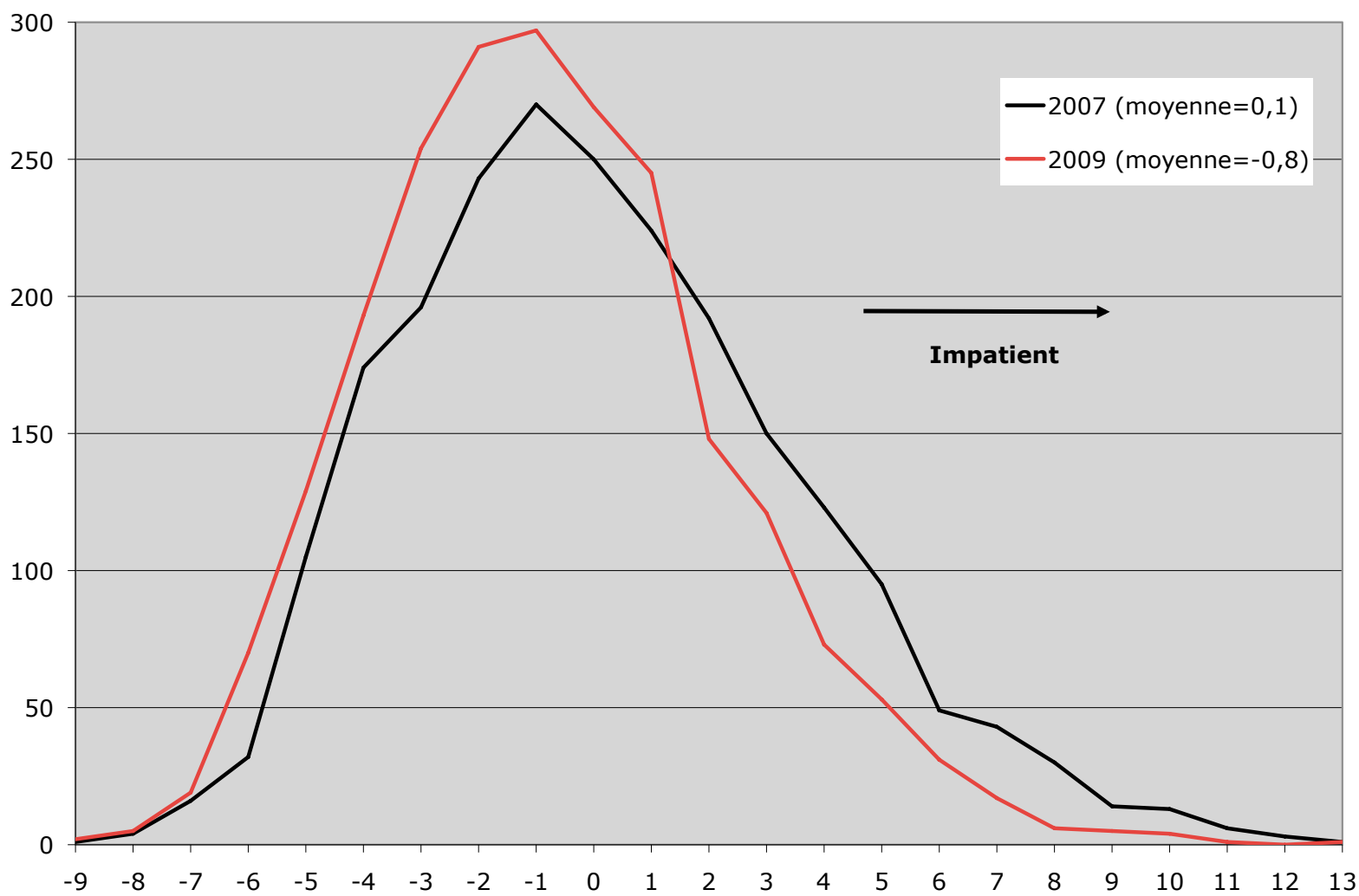

Source : TNS 2007-2009 (panel) 